\title{
DNA methylation screening after roux-en $Y$ gastric bypass reveals the epigenetic signature stems from genes related to the surgery per se
}

\author{
C. F. Nicoletti ${ }^{1}$, M. A. S. Pinhel ${ }^{1}$, A. Diaz-Lagares ${ }^{2,3}$, F. F. Casanueva ${ }^{4,5}$, A. Jácome ${ }^{6}$, V. C. Pinhanelli ${ }^{1}$, \\ B. A. P. de Oliveira ${ }^{1}$, A. B. Crujeiras ${ }^{4,5^{*}}$ and C. B. Nonino ${ }^{1 *}$
}

\begin{abstract}
Background/objectives: Obesity has been associated with gene methylation regulation. Recent studies have shown that epigenetic signature plays a role in metabolic homeostasis after Roux-en Y gastric bypass (RYGB). To conduct a genome-wide epigenetic analysis in peripheral blood to investigate whether epigenetic changes following RYGB stem from weight loss or the surgical procedure per se.

Subjects/methods: By means of the Infinium Human Methylation 450 BeadChip array, global methylation was analyzed in blood of 24 severely obese women before and 6 months after RYGB and in 24 normal-weight women (controls).

Results: In blood cells, nine DMCpG sites showed low methylation levels before surgery, methylation levels increased after RYGB and neared the levels measured in the controls. Additionally, $44 \mathrm{CpG}$ sites associated with the Wnt and p53 signaling pathways were always differently methylated in the severely obese patients as compared to the controls and were not influenced by RYGB. Finally, 1638 CpG sites related to inflammation, angiogenesis, and apoptosis presented distinct methylation in the post-surgery patients as compared to the controls.
\end{abstract}

Conclusion: Bariatric surgery per se acts on CpGs related to inflammation, angiogenesis, and endothelin-signaling. However, the gene cluster associated with obesity remains unchanged, suggesting that weight loss 6 months after RYGB surgery cannot promote this effect.

Keywords: Obesity, Bariatric surgery, Epigenetics, DNA methylation, Pathway, Weight loss

\section{Background}

There has been increasing evidence that obesity is not a simple consequence of unbalanced dietary habits or sedentary behaviors, but a systemic and complex disease resulting from body homeostasis and metabolism dysregulation $[1,2]$. In this sense, genetic approaches have demonstrated that obesity could be an inherited disease stemming from genetic background and heritable epigenetic factors [3].

\footnotetext{
* Correspondence: anabelencrujeiras@hotmail.com; carla@fmrp.usp.br ${ }^{4}$ Epigenomics in Endocrinology and Nutrition, Health Research Institute of Santiago (IDIS), University Clinical Hospital of Santiago (CHUS/SERGAS) and Santiago de Compostela University (USC), Santiago de Compostela, Spain 'Laboratory of Nutrigenomics Studies, Department of Internal Medicine, Ribeirão Preto Medical School, University of Sao Paulo, Sao Paulo, Brazil Full list of author information is available at the end of the article
}

In the context of epigenetics research, DNA methylation has been the most extensively studied phenomenon related to obese and metabolic phenotypes [4]. DNA methylation consists in addition of methyl $(-\mathrm{CH} 3)$ groups at CpG dinucleotides, which influences gene transcription [5]. Regarding predisposition to metabolic disorders, obesity has been associated with regulation of the methylation of numerous candidate genes [6]. Animal studies have shown that high-fat diet modifies the epigenetics and transcriptional activity of lipid homeostasis-related genes, which contributes to obesity development [7]. On the other hand, obesity-induced inflammation and oxidative stress due to fat accumulation exposes the genome of many tissues to several systemic factors, which can determine the DNA methylation profile [8]. Indeed, this 
abnormal global epigenetic state drives obesogenic expression patterns [9]. Therefore, alterations in the epigenome may cause molecular changes in pathways that are associated with obesity and may improve metabolic health after therapeutic intervention [10].

Concerning obesity treatments, bariatric surgery is the most effective intervention for severe obese. Roux-en $\mathrm{Y}$ gastric bypass (RYGB) is one of the most often performed techniques and corresponds to $43 \%$ of all bariatric surgery procedures [11, 12]. Although many mechanisms may contribute to weight loss and metabolic improvement after RYGB (e.g., decreased food ingestion, changes in gut hormones and peptide secretion, and nutrient disabsortion) $[13,14]$, recent studies have demonstrated that epigenetic signature plays a role in metabolic homeostasis after surgery $[15,16]$. Altered methylation of specific DNA sites has been verified after weight loss $[16,17]$ and bariatric surgery [18].

Knowing whether epigenetic alterations stem from weight and fat loss or the surgical procedure per se is important. Some methylation changes may result from obesity phenotype marks [4]; however, the DNA methylation profile can be a biological effect of calorie restriction [19]. Even after significant weight is lost and comorbidities are improved, patients submitted to RYGB remain with an obese profile at the early postoperative period [20]. Bariatric surgery can change the molecular pathways involved in inflammatory and immunological response, cell differentiation, and oxidative stress regulation [21], so we suggest that the surgical procedure itself may epigenetically modify obesity-independent genes.

In this study, we conduct a genome-wide epigenetic analysis in peripheral blood to investigate the role that RYGB plays in DNA methylation pattern changes. Also, we evaluate whether and to what magnitude DNA methylation profile changes are associated with the molecular pathogenic mechanism of weight loss and/or with the response pathways related to the surgical procedure.

\section{Methods}

\section{Subjects and phenotypic characteristics}

This is a prospective study involving adult female subjects from a mixed ethnicity population assisted at a public health service in Brazil. The subjects were divided into two groups: 1. RYGB Group: 24 severely obese women (Body mass index (BMI) $>35 \mathrm{~kg} / \mathrm{m}^{2}, 36.9 \pm 10.2$ years) submitted to bariatric surgery (RYGB technique), and 2 . Control Group or controls: 24 normal-weight women (BMI ranging from 18.5 to $24.9 \mathrm{~kg} / \mathrm{m} 2,36.9 \pm 11.8$ years). Men were excluded to avoid the possible biases due to the hormonal influences. Subjects belonging to the RYGB Group were selected from the Bariatric Surgery Outpatient Clinic of a university hospital and had no history of diabetes mellitus. Controls had not had any body weight changes in the previous three months. Patients submitted to the modified standard surgical technique (RYGB), who missed the service follow up, who were pregnant or lactating, and who had a history of alcohol or drug abuse were excluded. Participants were informed about the study protocol, and only those who agreed with its implementation were included.

The RYGB Group was evaluated before (baseline) and six months after bariatric surgery, the Control Group was evaluated only once. Anthropometric measurements (weight, height, BMI, and waist circumference (WC)), body composition analysis (fat mass and fat-free mass), and biochemical evaluation (glucose, total cholesterol, HDL cholesterol, LDL cholesterol, and triglycerides) were accomplished as described previously [22].

\section{DNA methylation analysis}

Blood for DNA methylation analysis was collected after fasting, according to standard procedures. DNA was extracted from peripheral blood leukocytes with the GE Health Care kit (Illustra blood genomic Prep Mini Spin kit), according to the supplier's instructions. DNA fragmentation or RNA contamination was analyzed by $1 \%$ agarose gel electrophoresis. DNA (500 ng) bisulfite was converted by using the EZ DNA methylation kit Methylation-Gold (Zymo Research, CA, USA), according to the manufacturer's instructions, which converted cytosine to uracil.

Methylation was analyzed with the Infinium Human Methylation 450 BeadChip array). High-quality treated DNA was hybridized to the Infinium Human Methylation 450 BeadChips (Illumina) following the Illumina Infinium HD methylation protocol. Beadchips were scanned with the Illumina HiScanSQ system, and image intensities were extracted with the Genome Studio (2011.1) Methylation Module (v1.8.5). Blood samples from each subject were hybridized to the same physical chip to minimize biases.

DNA quality checks, bisulfite modification, hybridization, data normalization, and statistical filter were performed as described elsewhere $[23,24]$. The methylation level was expressed as a beta $(\beta)$ value that was calculated as the intensity of the methylated channel divided by the total intensity $(\beta=\operatorname{Max}($ SignalB, 0) / $($ Max (SignalA, 0) + Max $($ SignalB, 0$)+100))$. $\beta$ values range from 0 (unmethylated) to 1 (fully methylated) and can be broadly interpreted as the percentage of CpG methylation. For genomic regions, methylation was calculated as the mean $\beta$ for all the probes located within the region annotated by Illumina: TSS200 (TSS - transcription start site), TSS1500, 5'UTR (UTR untranslated region), 1st Exon, gene body, 3'UTR, and intergenic. In addition, the probes that were considered single nucleotide polymorphisms were filtered out. The final amount of valid CpGs in this study was 476,895. 
Differential methylation analyses aimed to evaluate methylation differences between the study groups. To this end, the mean beta variation $(\Delta \beta)$ was calculated for a given CpG site by subtracting the mean beta value from the pool of pre-surgery samples (pre-surgery period) as compared to the pool of samples collected after RYGB (six months after surgery) or from the pool of pre-surgery samples as compared to the pool of samples from the Control Group.

\section{Statistical analysis}

The phenotypic characteristics of the subjects included in the study were expressed as mean values and standard deviation. Data normality was verified by the Shapiro-Wilk test. Paired t test was carried out to compare pre- and post-operative values, and independent $t$ test was used to compare the RYGB Group and the Control Group. Linear regression models were employed to test how the CpG methylation level affected the anthropometric and biochemical characteristics, adjusted for age. Also, Bonferroni's correction for multiple comparisons was applied. All the analyses were conducted with the Statistical Package software for Social Sciences (SPSS version 20.0, Inc. Chicago, IL), significance was set at $p<0.05$.

To identify consistent patterns of differentially methylated CpGs, parametric t test was accomplished. Sample size led us to apply t test instead of other methods that can adjust for confounding factors. Values of $p$ were adjusted for multiple comparisons by using the false discovery rate procedure described by Benjamini and Hochberg. In this analysis, a false discovery rate below $5 \%$ (q value) was considered statistically significant. However, given the sample size, raw $p$ values of 0.01 were selected as a less stringent cutoff for differential methylation than $\mathrm{q}$ values. Indeed, a threshold for the significant $\mathrm{CpG}$ sites based on $\Delta \beta$ with a minimum value of $5 \%$ (value greater than 0.05 or less than -0.05) was applied. Results were quite robust even though only individuals were evaluated in each group. These statistical analyses were performed with $\mathrm{R}$ software (version 3.2.0).

By crossing and comparing the differentially methylated CpG sites (DMCpGs) identified in two different periods (before and after RYGB) and in two study groups (pre-surgery patients versus controls and post-surgery patients versus controls), a Venn diagram was created (http://bioinfogp.cnb.csic.es/tools/venny/). Hierarchical cluster analysis of the significant CpGs was carried out with Heatmap function and Genome Studio (2011.1).

To gain even better understanding of the biological relevance of the significant associations between DNA methylation and the studied phenotypes, a hypergeometric test was conducted for the biological processes defined by gene ontology (GO) [25]. This evaluation identified the significant over-representation of GO terms in our lists of selected genes with respect to the other for the entire genome. The IDs were loaded and analyzed against the human reference genome by means of a false discovery rate threshold of $p<0.05$.

\section{Results}

\section{Phenotypic characteristics}

The severely obese patients had higher weight, BMI, WC, percentage of fat mass (\%FM), triglycerides, and total cholesterol as well as lower plasma HDL cholesterol than the controls. RYGB significantly reduced weight, BMI, WC, percentage of fat- free mass, \%FM, and serum glucose and lipid levels, but it did not change HDL cholesterol. However, the post-surgery patients still presented anthropometric measurements that are characteristic of obesity, but their lipid profiles (except HDL cholesterol) resembled the lipid profiles of the controls (Additional file 1).

\section{Methylation data}

All results are summarized in Additional file 3. After normalization, we found 476,895 final valid CpG sites and pre, posoperative and normal weight results are present at below topics. Raw data is in Additional file 4.

\section{Identification of different methylated CpGs between groups and surgery times}

Comparison between the methylation profiles of the pre-surgery patients and the controls revealed 1074 DMCpG sites that were related to 769 unique genes. Significant differences between the groups ranged from 0.05 to 0.27 (from 5 to 27\%). Even though there was no statistical difference between the average DNA methylation levels of the 1074 DMCpG sites $(0.42 \pm 0.26$ versus $0.41 \pm 0.28, p=$ 0.168), the majority of the CpG sites (66.2\%) showed higher methylation in the pre-surgery patients, especially in the TSS200 region and gene island (Additional file 2).

Additionally, RYGB elicited changes in 666 CpG sites located in 495 unique genes. Significant differences between the pre- and post-surgery periods ranged from 0.05 to 0.10 (from 5 to $10 \%$ ), and the average DNA methylation levels of all the DMCpG sites increased after surgery (from $0.44 \pm 0.13$ to $0.49 \pm 0.12, p<0.001$ ). These results showed higher methylation of these CpG sites after RYGB.

Comparison between the post-surgery patients and the controls detected 3223 DMCpG sites (2065 unique genes). Significant differences between the groups ranged from 0.05 to 0.31 (from 5 to $31 \%$ ). Indeed, the average DNA methylation levels of the $3223 \mathrm{DMCpG}$ sites were greater in post-surgery patients $(0.49 \pm 0.18$ versus $0.45 \pm 0.19, p<0.001)$.

\section{Identification of target genes specifically associated with body weight changes}

Comparison between DMCpG sites in the pre-surgery patients versus the controls and in the pre-surgery period 
versus the post-surgery period by means of a Venn diagram showed that nine CpGs located in nine different genes were common in both analyses (Fig. 1a). These CpGs exhibited lower methylation levels in the pre-surgery e patients as compared to the controls, after RYGB, the methylation levels increased and neared the levels verified in the controls (Fig. 1b). Table 1 lists the nine CpGs associated with body weight changes as revealed by this study. The largest difference was observed for $\operatorname{cg} 04789056$, located in the chromosome 14 open reading frame 93 (C14orf93) gene ($13 \%$ in the pre-surgery patients as compared to the controls). This was also the CpG that changed the most after RYGB $(+13 \%$ in the post-surgery period as compared to the preoperative period). Figure $1 \mathrm{c}$ indicates the gene functions, which include DNA and RNA binding, protein complex binding, and receptor ligand activity.

\section{Identification of genes specific for obesity status}

The Venn diagram depicting the DMCpG sites found in the pre-surgery patients versus the controls and in the post-surgery period versus the pre-surgery period showed that the severely obese patients and the controls always had 544 different sites, located in 386 unique genes (Fig. 2a). These CpG sites were different in the controls and were not influenced by the surgical procedure (Fig. $2 \mathrm{~b}$ ). Table 2 summarizes the top $20 \mathrm{CpG}$ sites, which were always differentially methylated in the severely obese patients as compared to the controls. Among the encoded genes, NADH:ubiquinone oxidoreductase subunit S6 (NDUFS6) and mitochondrial ribosomal protein L36 (MRPL36) were the genes that were the most represented with two DMCpG sites located in the island.

$\mathrm{GO}$ analysis helped to investigate the potential biological relevance of the genes with different DNA methylation status in the severely obese patients and the controls (Fig. 2c). Regarding biological processes, most of the differentially methylated genes were associated with transcription regulation, signal transduction, apoptosis, transport, and cell adhesion. Interestingly, pathway analysis identified that most of the genes were related to the Wnt and p53 signaling pathways (Fig. 2c).

\section{Identification of genes related to the effect of bariatric surgery per se}

According to evidence gathered herein, $2678 \mathrm{CpG}$ sites were not statistically different in the severely obese patients and the controls, however, these genes became differentially methylated after RYGB. These DMCpG sites
A Venn diagram of $\mathrm{CpGs}$ involved with weight changes

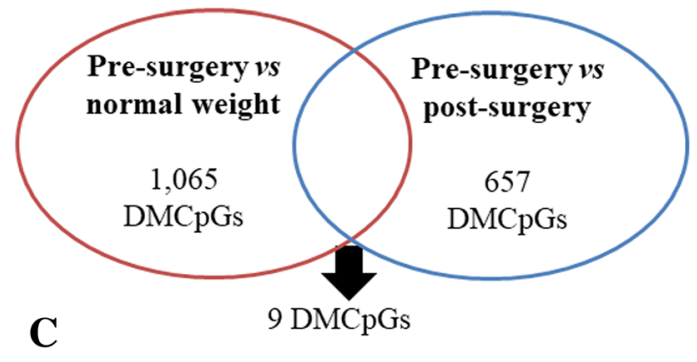

Genes

C14orf93 RNA binding

ZP3 Receptor ligand activity

TRIO ATP binding

SPEG Protein complex binding

C1orf189 Protein binding

C1orf43

FOXN3

CYFIP1

MYOMI
B Heatmap of CpGs involved with weight changes

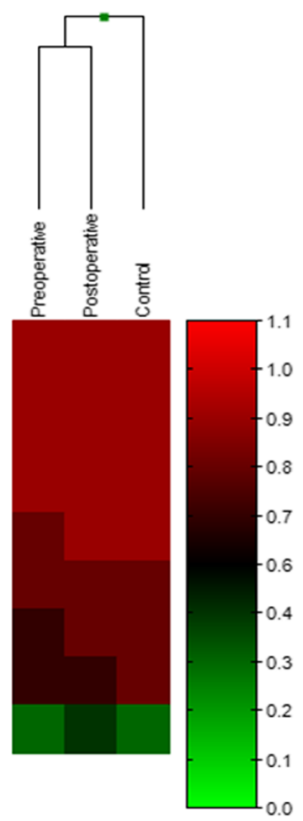

Fig. 1 Clustering analysis of the differentially methylated CpG sites. a: Venn diagram of the DMCpG sites detected in the pre-surgery patients versus the controls and in the pre-surgery period versus the post-surgery period. Nine common sites indicated genes related to body weight changes. b: Supervised clustering of the nine CpGs that were differentially methylated in the pre-surgery patients as compered to the controls and which had their methylation profiles altered after the surgical procedure. c: Gene function of eight genes represented by the nine differentially methylated $\mathrm{CpG}$ sites 


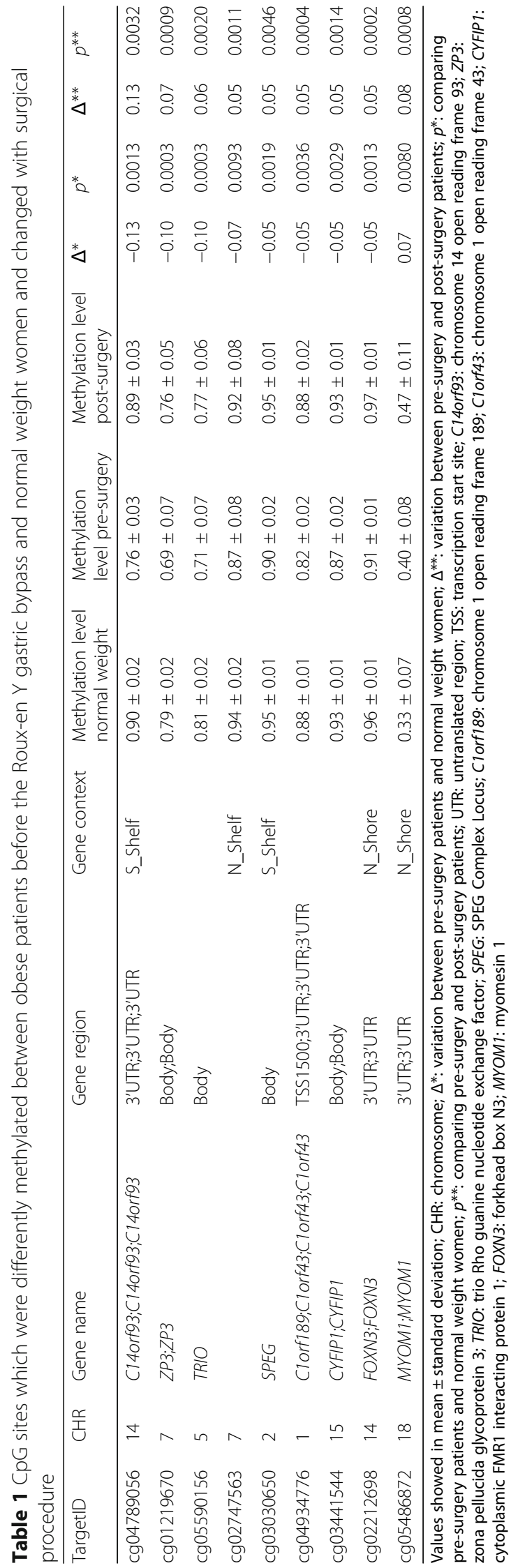




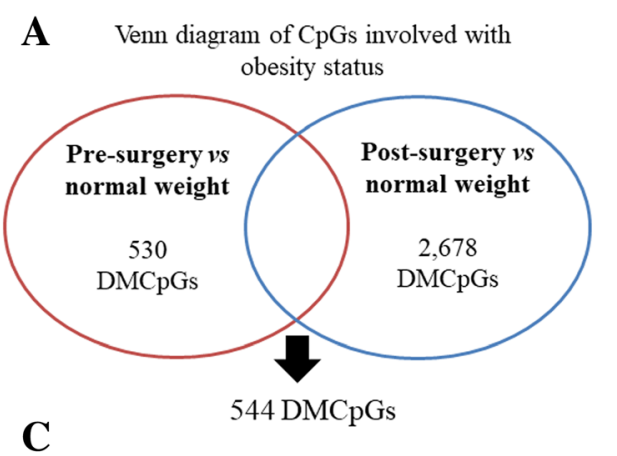

B Heatmap of CpGs involved with obesity status

Gene ontology of CpGs involved with obesity status
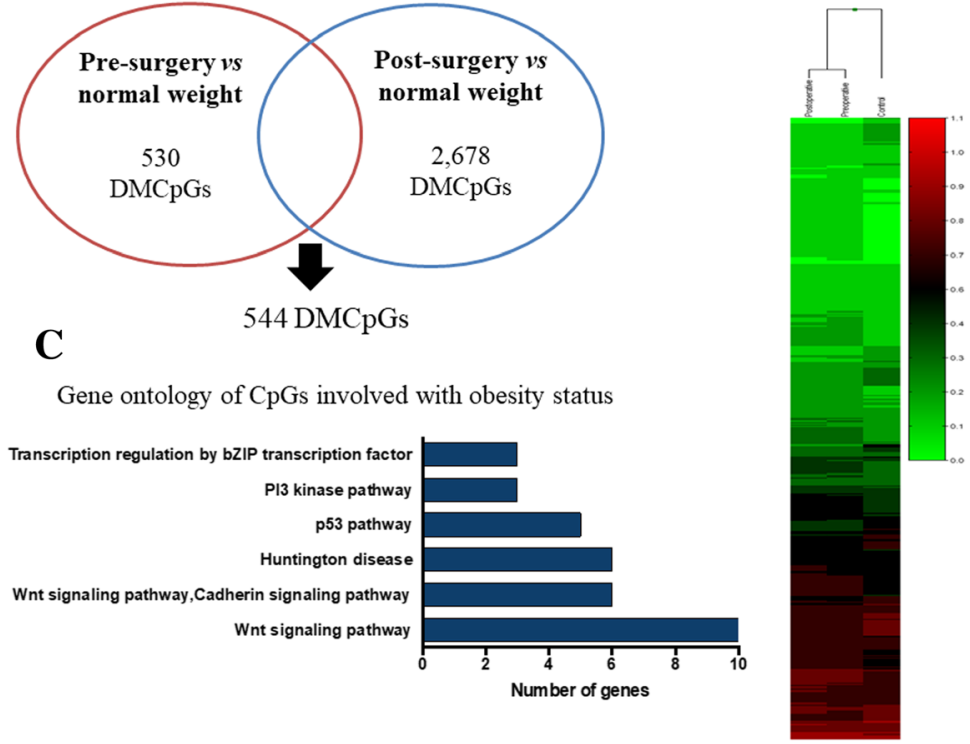

D Heatmap of CpGs associated with surgery effect per se

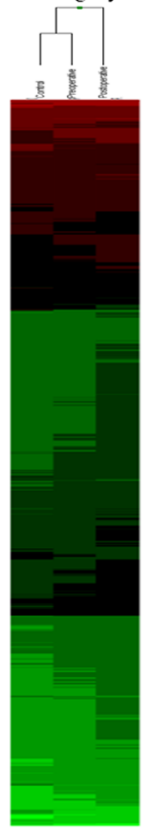

E Gene ontology of CpGs associated with surgery effect per se

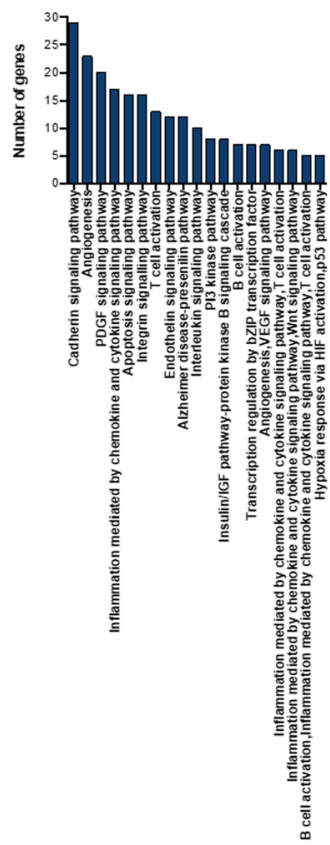

Fig. 2 Clustering analysis of the differentially methylated $\mathrm{CpG}$ sites. a: Venn diagram of the DMCpG sites in the pre-surgery patients as compared to the controls and in the post-surgery patients as compared to the controls. A total of 544 common sites were associated with obesity status, and 2678 DMCpG sites were related to an effect of bariatric surgery per se. b: Supervised clustering of the 544 CpGs that were differentially methylated in the pre-surgery patients as compared to the controls. c: Summary of the gene ontology (GO) analysis of the biological process categories for the 386 genes represented by the 544 differentially methylated CpG sites. d: Supervised clustering of the 2678 CpGs that were differentially methylated in the post-surgery patients as compared to the controls. e: Summary of the gene ontology (GO) analysis of the biological process categories for the 1638 genes represented by the 2678 differentially methylated CpG sites

were located in 1638 genes, most of the sites (2219 CpGs) showed high methylation after RYGB (Fig. 2d). Table 3 depicts the top 20 CpG sites that were differently methylated in the post-surgery patients as compared to the controls. The most significant difference was observed for cg07875360 in the NDUFS6 and MRPL36 genes $(+35 \%$ in the controls as compared to the post-surgery patients). 


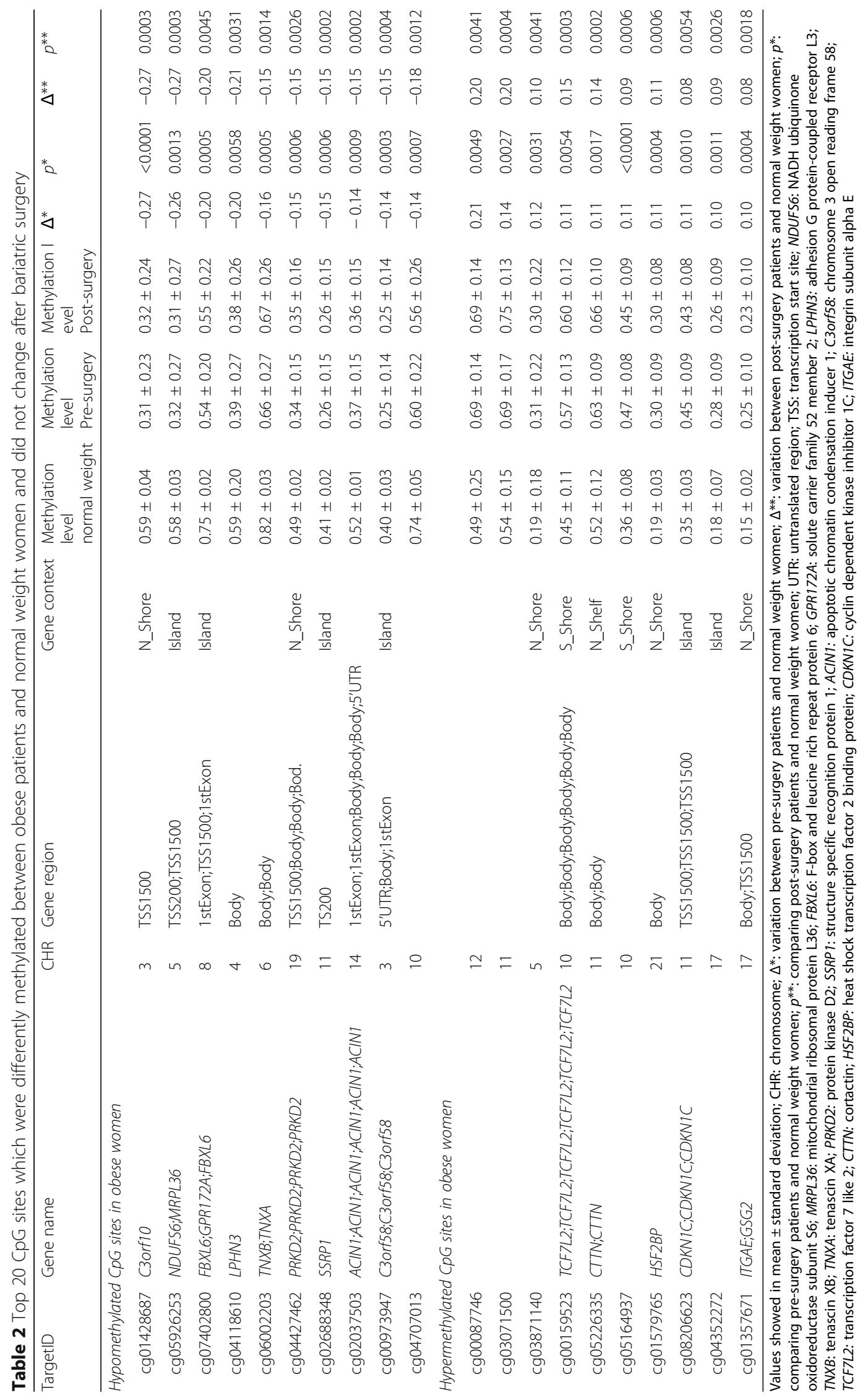


Table 3 Top 20 CpG sites that became differently methylated from control group after Roux-en Y gastric bypass

\begin{tabular}{|c|c|c|c|c|c|c|c|c|}
\hline TargetID & Gene name & $\mathrm{CHR}$ & Gene region & Gene context & $\begin{array}{l}\text { Methylation level } \\
\text { normal weight }\end{array}$ & $\begin{array}{l}\text { Methylation level } \\
\text { Post-surgery }\end{array}$ & $\Delta$ & $p$ \\
\hline \multicolumn{9}{|c|}{ Hypomethylated CpG sites in obese women after RYGB } \\
\hline $\operatorname{cg} 07875360$ & NDUFS6;MRPL36 & 5 & TSS200;TSS1500 & Island & $0.72 \pm 0.03$ & $0.40 \pm 0.32$ & -0.31 & 0.0011 \\
\hline cg01759136 & & 6 & & & $0.68 \pm 0.04$ & $0.40 \pm 0.30$ & -0.28 & 0.0034 \\
\hline cg00554442 & LMF1 & 16 & TSS200 & Island & $0.26 \pm 0.01$ & $0.01 \pm 0.01$ & -0.25 & 0.0046 \\
\hline $\operatorname{cg} 05444312$ & HIST1H2BM;HIST1H2AJ & 6 & TSS200;TSS200 & S_Shore & $0.40 \pm 0.04$ & $0.21 \pm 0.19$ & -0.19 & 0.0009 \\
\hline cg07962043 & TMEM132A;TMEM132A & 11 & TSS1500;TSS1500 & N_Shore & $0.38 \pm 0.02$ & $0.25 \pm 0.11$ & -0.13 & $<0.0001$ \\
\hline cg04450994 & SLC22A23;SLC22A23 & 6 & Body;Body & & $0.56 \pm 0.06$ & $0.45 \pm 0.08$ & -0.11 & 0.0003 \\
\hline cg01035945 & ZNF323;ZNF323;ZKSCAN3 & 6 & 5'UTR;5'UTR;TSS1500 & & $0.25 \pm 0.03$ & $0.14 \pm 0.12$ & -0.11 & 0.0054 \\
\hline cg07929642 & ANKRD11 & 16 & $5^{\prime} U T R$ & & $0.75 \pm 0.05$ & $0.64 \pm 0.08$ & -0.11 & 0.0006 \\
\hline cg06115576 & $D I P 2 C$ & 10 & Body & & $0.86 \pm 0.02$ & $0.75 \pm 0.18$ & -0.11 & $<0.0001$ \\
\hline cg00510787 & C6orf211;RMND1 & 6 & TSS1500;5'UTR & N_Shore & $0.29 \pm 0.03$ & $0.18 \pm 0.13$ & -0.11 & 0.0097 \\
\hline \multicolumn{9}{|c|}{ Hypermethylated $C p G$ sites in obese women after RYGB } \\
\hline cg07275179 & ATXN7;ATXN7 & 3 & Body;Body & & $0.41 \pm 0.09$ & $0.53 \pm 0.11$ & 0.12 & 0.0002 \\
\hline cg07212327 & FAM49B & 8 & $5^{\prime} U T R$ & N_Shelf & $0.50 \pm 0.09$ & $0.62 \pm 0.10$ & 0.12 & $<0.0001$ \\
\hline cg07401324 & PTPRJ;PTPRJ & 11 & Body;Body & & $0.35 \pm 0.07$ & $0.48 \pm 0.09$ & 0.12 & 0.0002 \\
\hline cg02353916 & LOC285550 & 4 & $3^{\prime} U T R$ & & $0.44 \pm 0.10$ & $0.57 \pm 0.10$ & 0.13 & $<0.0001$ \\
\hline cg00123214 & RWDD3;RWDD3 & 1 & Body;Body & S_Shelf & $0.52 \pm 0.23$ & $0.65 \pm 0.15$ & 0.13 & 0.0058 \\
\hline cg02387226 & UNG;UNG & 12 & TSS1500;TSS1500 & N_Shore & $0.52 \pm 0.02$ & $0.65 \pm 0.03$ & 0.13 & 0.0069 \\
\hline cg03494429 & & 12 & & & $0.64 \pm 0.07$ & $0.79 \pm 0.06$ & 0.14 & 0.0093 \\
\hline cg04412904 & & 16 & & & $0.22 \pm 0.19$ & $0.37 \pm 0.23$ & 0.15 & 0.0028 \\
\hline cg07093060 & & 3 & & N_Shelf & $0.66 \pm 0.29$ & $0.84 \pm 0.10$ & 0.18 & 0.0082 \\
\hline cg07572984 & & 14 & & & $0.60 \pm 0.30$ & $0.80 \pm 0.20$ & 0.20 & 0.0013 \\
\hline
\end{tabular}

Values showed in mean \pm standard deviation; CHR: chromosome; $\Delta$ : variation between post-surgery patients and normal weight women; $p$ : comparing postsurgery patients and normal weight women UTR: untranslated region; TSS: transcription start site; NDUFS6: NADH:ubiquinone oxidoreductase subunit S6; MRPL36: mitochondrial ribosomal protein L36; LMF1: lipase maturation factor 1; HIST1H2BM: histone cluster 1 H2B family member m; HIST1H2AJ: histone cluster 1 H2A family member j; TMEM132A: transmembrane protein 132A; SLC22A23: solute carrier family 22 member 23; ZNF323: zinc finger and SCAN domain containing 31; AN3: tripartite motif containing 44; ANKRD11: ankyrin repeat domain 11; DIP2C: disco interacting protein 2 homolog C; C6orf211: acidic residue methyltransferase 1; RMND1: required for meiotic nuclear division 1 homolog; ATXN7: ataxin 7; FAM49B: family with sequence similarity 49 member B; PTPRJ: protein tyrosine phosphatase, receptor type J; RWDD3: RWD domain containing 3; UNG: uracil DNA glycosylase

GO analysis helped to evaluate the biological processes and pathways of the genes with DMCpG sites in the post-surgery patients and the controls. Among the biological processes, transcription regulation, signal transduction, cell adhesion, blood coagulation, apoptotic process, ion transport, and protein phosphorylation exhibited the majority of genes. Indeed, pathway analysis identified that the genes were related to cadherin signaling, angiogenesis, apoptosis, inflammation, and interleukin pathways (Fig. 2e).

\section{Epigenetic signatures and phenotypic characteristics}

Among the nine genes related to body weight changes and the top $20 \mathrm{CpG}$ sites associated with obesity status, the methylation levels of the myomesin 1 (MYOM1), transmembrane protein 48 (TMEM48), and heat shock transcription factor 2 binding protein (HSF2BP) genes were associated with the anthropometric and biochemical features (Fig. 3). Pre-surgery patients with higher cg05486872 methylation (located in the MYON1 gene) had greater BMI. Moreover, cg00959749 located in the TMEM48 gene was always differentially methylated in the severely obese patients as compared to the controls, which indicated that this methylation was positively correlated (baseline level) with the percentage of weight loss and BMI change. This CPG site presented low methylation levels in the severely obese patients. The patients with greater methylation levels at this site lost more weight. Another $\mathrm{CpG}$ was also associated with changes in lipid profile after the surgical procedure: $\operatorname{cg} 01579765$ in the HSF2BP gene was positively correlated with reduced cholesterol and LDL concentrations. The effect of methylation levels on these phenotypic characteristics remained apparent after regression adjusted by age.

\section{Discussion}

Genome-wide DNA methylation analysis identified CpG sites that are specific for obesity per se after bariatric 

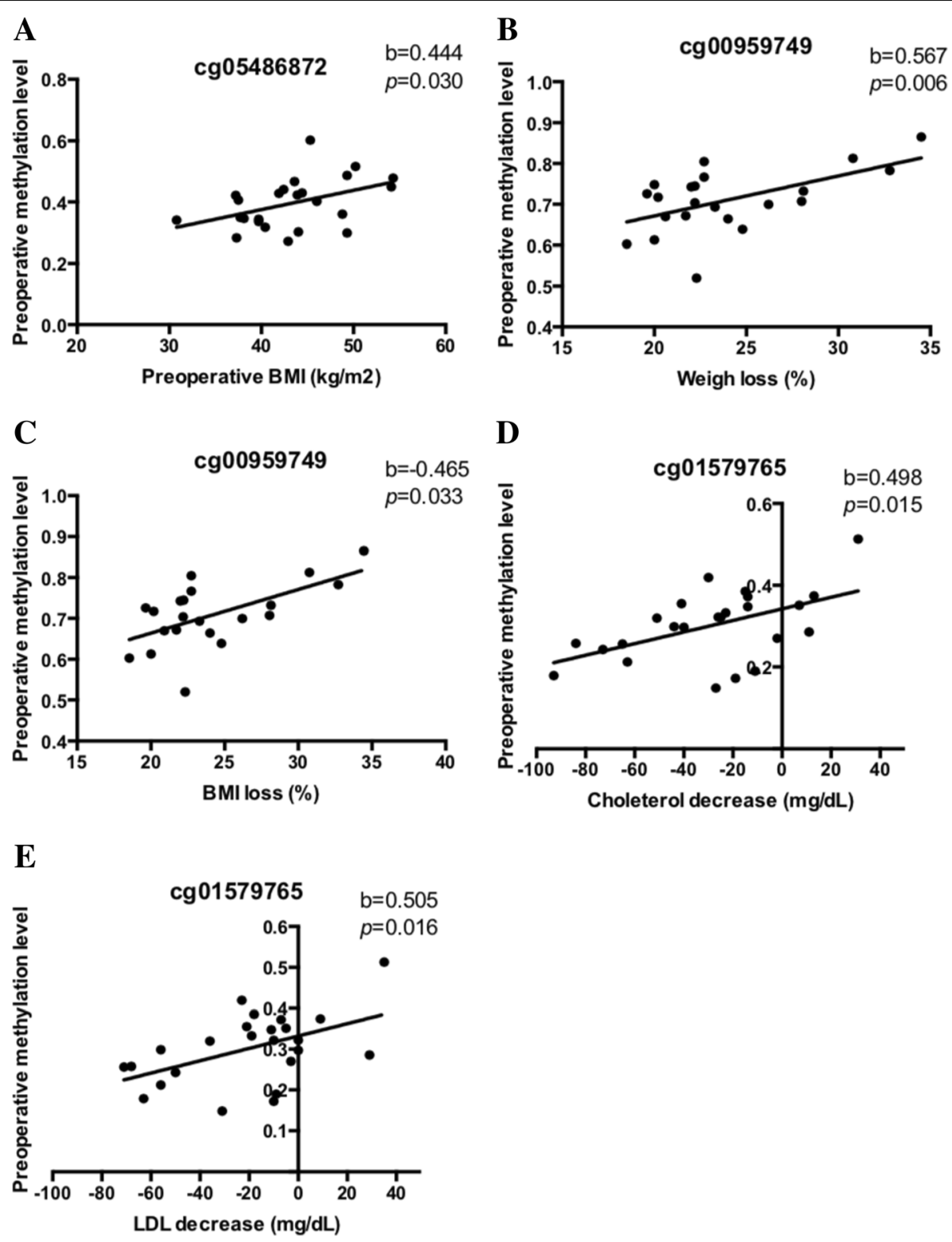

Fig. 3 Linear regression models adjusted by age showing the positive effect of baseline DNA methylation on the phenotypic characteristics. a Effect of the MYOM1 gene on BMI. $\mathbf{b}$ Effect of the TMEM48 gene on the percentage of weight loss. $\mathbf{c}$ Effect of the TMEM48 gene on the percentage of BMI change. $\mathbf{d}$ Effect of the HSF2BPgene on cholesterol decrease. e Effect of the HSF2BP gene on LDL-cholesterol decrease. BMI: body mass index. LDL: low-density lipoprotein

surgery, the identified sites remained different in the operated patients as compared to the controls. This analysis also identified CpG sites that are modified by bariatric surgery-induced weight loss as well as CpG sites that have their methylation levels specifically modified by RYGB irrespective of their association with the obesity status.

The obesity pathophysiology is strongly associated with adipose tissue dysregulation and epigenetic changes are more tissue specific. This fact represents a huge challenge to evaluate epigenetic mechanisms in longitudinal studies because adipose tissue is an inaccessible tissue without surgery. Instead of adipose tissue biopsies, peripheral blood cells are frequently used for epigenetic analysis. In this regard, very recently it was demonstrated that epigenetic biomarkers in blood can mirror epigenetic signatures in biologically relevant target tissues such as adipose tissue [26-28]. These previous results suggest that the assessment of DNA methylation in whole blood can identify robust and biologically relevant epigenetic variation. Strikingly, we were able to identify epigenetic markers associated with adipose tissue and inflammation (obesity pathogenesis) in blood leukocytes. 
The identified epigenetic signature could be relevant to the personalized management of obesity, mainly after bariatric surgery, aiming for better result of weight loss.

The scientific literature contains extensive description of the advantages of bariatric surgery over acute significant weight loss in terms of the improvement in comorbidities $[29,30]$. Nevertheless, little is known about the epigenetic mechanisms associated with the metabolic and clinical benefits provided by bariatric surgery. The present study showed that RYGB promoted changes in $666 \mathrm{CpG}$ sites. Previous evidence suggests that changes in whole blood DNA methylation may be related to body weight and fasting plasma glucose reduction [31]. Other studies have described epigenetic changes in the adipose tissue [32, 33] and skeletal muscle [34] after RYGB.

In this context, we were able to identify 544 DMCpG sites related to obesity status in leukocytes. Interestingly, these sites remained differentially methylated in obese and normal-weight women irrespective of the bariatric surgery effect on weight loss. The main routes associated with these sites were the Wnt and p53 signaling pathways, which are associated with adipocyte differentiation $[35,36]$ and lipid/insulin resistance metabolism [37, 38], respectively. We hypothesized that the weight loss reached by the operated patients six months after surgery was not able to modify this epigenetic profile because the patients remained obese at this time. Based on our analysis, only nine CpG sites were specifically related to body weight changes, which highlighted genes involved in obesity, adipogenesis, and hepatic glucose utilization. These data suggested that the genes associated with these CpG sites should be investigated as new and specific targets of obesity and weight loss in future studies.

On the other hand, bariatric surgery promoted epigenetic regulation of several genes, which seemed to be an effect of the surgery per se because their differential methylation was not associated with the obesity status. These CpG sites encoded mainly genes related to angiogenesis, inflammation, and endothelin-signaling pathways. This could be explained by the fact that the surgery itself was an invasive procedure that promoted cell damage and inflammation, with consequent tissue regeneration. Moreover, our previous findings had shown distinct changes in the methylation profile of inflammatory genes after different obesity treatments, with reduction in the $I L-6$ methylation level six months after RYGB [18].

Little is discussed about the effects that the different surgical techniques may have on the DNA methylation profile. Knowing that biochemical (bile acids, cholesterol, glycemia) and metabolic (ie improvement of insulin resistance, dyslipidemia, inflammation, oxidative stress) changes due to bariatric surgery may alter the DNA methylation profile of several genes [39] and that the different surgical techniques (restrictive, disabsorptive or mixed) cause diverse nutritional and metabolic changes [40] it is of great value the comparison of the bariatric techniques. In this context, authors found no difference in the methylation of the interspersed nucleotide element 1 (LINE-1) in patients underwent Roux-en-Y gastric bypass or laparoscopic sleeve gastrectomy [41].

Nowadays researches are made in the identification of specific biomarkers predicting the response to RYGB procedure [42]. As an example, a previous study by our research group identified difference between the baseline SERPINE-1 methylation of the individual who lost more or less weight after bariatric surgery [43]. On the other hand, other authors recently found no association between the methylation level of food intake-related genes and the response to surgery [42]. In line of this, linear regression analysis between the baseline methylation levels and the phenotypic markers were performed and showed association of the MYOM1, TMEM48, and $H S F 2 B P$ methylation levels with the anthropometric and metabolic parameters. Despite the scarce literature data on this topic, different MYOM1 gene expression has been detected in human skeletal muscle cells of obese and lean subjects [44]. Furthermore, a study evaluating the adipose tissue of obese patients submitted to a six-month caloric restriction intervention showed different TMEM48 expression between high and low responders to dieting [19].

Studies on epigenetic patterns have become highly important due to their plasticity in the face of external factors and to the individual's own response. Better understanding of the pathways altered by bariatric surgery will aid the development of new biomarkers and therapies for obesity treatment $[39,45]$.

The strength of this study lies on its longitudinal design, which allowed us to evaluate whether changes in the methylation profiles were due to surgical intervention and/or body weight loss. Although the observed differences were statistically significant, the magnitude of DNA methylation differences between the pre- and the post-surgery periods may be considered small. A possible explanation for this small magnitude would be the reduced period of postoperative evaluation.

\section{Conclusion}

RYGB promoted epigenetic changes in specific pathways, mainly the pathways related to inflammation, angiogenesis, and endothelin-signaling. Genome-wide DNA methylation analysis revealed that gene clusters remained unchanged even after bariatric surgery, suggesting that, despite the strong magnitude of the weight loss achieved by the patients 6 months after bariatric surgery, reaching a normal body weight may be necessary to revert the methylation profile associated with obesity. 


\section{Additional files}

Additional file 1: Table. Anthropometry, body composition and biochemical data of women who underwent Roux in $\mathrm{Y}$ gastric bypass and normal weight controls. (DOCX $19 \mathrm{~kb}$ )

Additional file 2: Figure. Gene regions of differential methylated CpG sites. (DOCX 199 kb)

Additional file 3: Figure. Diagram of differential methylated $\mathrm{CpG}$ sites founded in present study. (TIF $58 \mathrm{~kb}$ )

Additional file 4: Raw data. Datasets generated and/or analyzed during the current study. (XLSB 395235 kb)

\section{Abbreviations}

BMI: Body Mass Index; DMCpGs: Differentially methylated CpG sites; GO: Gene ontology; RYGB: Roux-en Y gastric bypass; TSS: Transcription start site; UTR: Untranslated region; WC: Waist circumference

\section{Acknowledgements}

Authors thank Andrea Gonzalez-Izquierdo, María Amil and Maribel Rendo from the department of Molecular and Cellular Endocrinology of IDIS for their support of research data management.

\section{Funding}

This study's data collection, DNA and statistical analysis was supported by São Paulo Research Foundation (FAPESP) (grants \#2017/07220-7, \#2016/05638-1 and \#2015/18669-0). Also, statistical and bioinformatics analysis was supported by "Centro de Investigacion Biomedica En Red" (CIBERobn) and grants (PI17/01287) from the "Instituto de Salud Carlos III" (ISCIII), Spain, co-financed by the European Regional Development Fund (FEDER), MINECO grants MTM2014-52876-R and MTM2017-82724-R, and by the Xunta de Galicia (Grupos de Referencia Competitiva ED431C-2016-015 and Centro Singular de Investigación de Galicia ED431G/01), all of them through the ERDF. A Diaz-Lagares is funded by a research contract "Juan Rodés" (JR17/00016) and Ana B Crujeiras is funded by a research contract "Miguel Servet" (CP17/00088) from the ISCIII, co-financed by the European Regional Development Fund (FEDER).

\section{Availability of data and materials}

All data generated or analysed during this study are included in this published article [and its supplementary information files].

\section{Authors' contributions}

CFN, FFC, ABC and CBN received funding, conceived and jointly supervised the project. MASP, BAPO and CNF coordinated the sample collection and clinical annotation. CFN and MASP performed the DNA methylation analysis. AJ conduced bioinformatic analysis. CFN, CBN, FFC, ABC and APB wrote the manuscript. CNF, $A D L$ and $A B C$ were involved in figure generation for the manuscript. CFN, ADL and $A B C$ performed the interpretation of the genome data. All authors read and approved the final manuscript.

\section{Ethics approval and consent to participate}

The study was conducted with the approval of the Hospital Ethics Committee and in agreement with the Declaration of Helsinki. Informed consent was obtained from all individual participants included in the study.

\section{Consent for publication}

Not applicable.

\section{Competing interests}

The authors declare that they have no competing interests.

\section{Publisher's Note}

Springer Nature remains neutral with regard to jurisdictional claims in published maps and institutional affiliations.

\section{Author details}

'Laboratory of Nutrigenomics Studies, Department of Internal Medicine, Ribeirão Preto Medical School, University of Sao Paulo, Sao Paulo, Brazil.
${ }^{2}$ Cancer Epigenomics, Translational Medical Oncology (Oncomet), Health Research Institute of Santiago (IDIS), University Clinical Hospital of Santiago (CHUS/SERGAS), Santiago de Compostela, Spain. ${ }^{3}$ Centro de Investigacion Biomedica en Red Cancer (CIBERONC), Madrid, Spain. ${ }^{4}$ Epigenomics in Endocrinology and Nutrition, Health Research Institute of Santiago (IDIS), University Clinical Hospital of Santiago (CHUS/SERGAS) and Santiago de Compostela University (USC), Santiago de Compostela, Spain. ${ }^{5} \mathrm{CIBER}$ Fisiopatologia de la Obesidad y Nutricion (CIBERobn), Madrid, Spain. ${ }^{6}$ Department of Mathematics, MODES group, CITIC, Universidade da Coruña, Faculty of Science, A Coruña, Spain.

Received: 27 August 2018 Accepted: 7 May 2019

Published online: 27 May 2019

\section{References}

1. Speakman JR, O'Rahilly S. Fat: an evolving issue. Dis Model Mech. 2012;5:569-73.

2. DeBoer MD. Obesity, systemic inflammation, and increased risk for cardiovascular disease and diabetes among adolescents: a need for screening tools to target interventions. Nutrition. 2013;29:379-86.

3. Campion J, Milagro Fl, Martinez JA. Individuality and epigenetics in obesity. Obes Rev. 2009;10:383-92.

4. van Dijk SJ, Molloy PL, Varinli H, Morrison JL, Muhlhausler BS, Members of epi S. Epigenetics and human obesity. Int J Obes. 2015;39:85-97.

5. Fan S, Zhang X. CpG island methylation pattern in different human tissues and its correlation with gene expression. Biochem Biophys Res Commun. 2009;383:421-5.

6. Crujeiras $A B$, Casanueva FF. Obesity and the reproductive system disorders: epigenetics as a potential bridge. Hum Reprod Update. 2015;21:249-61.

7. Zhang P, Chu T, Dedousis N, Mantell BS, Sipula I, Li L, Bunce KD, et al. DNA methylation alters transcriptional rates of differentially expressed genes and contributes to pathophysiology in mice fed a high fat diet. Mol Metab. 2017:6:327-39.

8. Demerath EW, Guan W, Grove ML, Aslibekyan S, Mendelson M, Zhou YH, et al. Epigenome-wide association study (EWAS) of BMI, BMI change and waist circumference in African American adults identifies multiple replicated loci. Hum Mol Genet. 2015:24:4464-79.

9. Youngson NA, Morris MJ. What obesity research tells us about epigenetic mechanisms. Philos Trans R Soc Lond Ser B Biol Sci. 2013;368:20110337.

10. Kirchner H, Sinha I, Gao H, Ruby MA, Schonke M, et al. Altered DNA methylation of glycolytic and lipogenic genes in liver from obese and type 2 diabetic patients. Mol Metab. 2016;5:171-83.

11. Hao Z, Mumphrey MB, Morrison CD, Munzberg H, Berthoud HR. Does gastric bypass surgery change body weight set point? Int J Obes Suppl. 2016;6:S37-43.

12. Schauer PR, Nor Hanipah Z, Rubino F. Metabolic surgery for treating type 2 diabetes mellitus: now supported by the world's leading diabetes organizations. Cleve Clin J Med. 2017:84:S47-56.

13. Falken $Y$, Hellstrom PM, Holst JJ, Naslund E. Changes in glucose homeostasis after roux-en-Y gastric bypass surgery for obesity at day three, two months, and one year after surgery: role of gut peptides. J Clin Endocrinol Metab. 2011:96:2227-35.

14. Rubino F, Forgione A, Cummings DE, Vix M, Gnuli D, Mingrone G, et al. The mechanism of diabetes control after gastrointestinal bypass surgery reveals a role of the proximal small intestine in the pathophysiology of type 2 diabetes. Ann Surg. 2006;244:741-9.

15. Quarta C, Schneider R, Tschop MH. Epigenetic ON/OFF switches for obesity. Cell. 2016;164:341-2

16. van Dijk SJ, Tellam RL, Morrison JL, Muhlhausler BS, Molloy PL. Recent developments on the role of epigenetics in obesity and metabolic disease. Clin Epigenetics. 2015:7:66.

17. Crujeiras AB, Campion J, Diaz-Lagares A, Milagro Fl, Goyenechea E, Abete I, et al. Association of weight regain with specific methylation levels in the NPY and POMC promoters in leukocytes of obese men: a translational study. Regul Pept R. 2013;186(1-6).

18. Sala P, de Miranda Torrinhas RSM, Fonseca DC, Ravacci GR, Waitzberg DL, Giannella-Neto D. Tissue-specific methylation profile in obese patients with type 2 diabetes before and after Roux-en-Y gastric bypass. Diabetol Metab Syndr. 2017;22;9:15.

19. Bouchard L, Rabasa-Lhoret R, Faraj M, Lavoie ME, Mill J, Perusse L, et al. Differential epigenomic and transcriptomic responses in subcutaneous adipose tissue between low and high responders to caloric restriction. Am J Clin Nutr. 2010;91:309-20. 
20. de Oliveira BAP, de Souza Pinhel MA, Nicoletti CF, de Oliveira CC, Quinhoneiro DCG, Noronha NY, et al. UCP2 and PLIN1 expression affects the resting metabolic rate and weight loss on obese patients. Obes Surg. 2017;27:343-8.

21. Pinhel MAS, Noronha NY, Nicoletti CF, de Oliveira BAP, Cortes-Oliveira C, Pinhanelli VC, et al. Changes in global transcriptional profiling of women following obesity surgery bypass. Obes Surg. 2018;28:176-86.

22. Nicoletti CF, de Oliveira AP, Brochado MJ, de Oliveira BP, Pinhel MA, Marchini JS, et al. UCP1 -3826 a>G polymorphism affects weight, fat mass, and risk of type 2 diabetes mellitus in grade III obese patients. Nutrition. 2016;32:83-7.

23. Sandoval J, Heyn H, Moran S, Serra-Musach J, Pujana MA, Bibikova M, et al. Validation of a DNA methylation microarray for 450,000 CpG sites in the human genome. Epigenetics. 2011;6:692-702.

24. Heyn H, Carmona FJ, Gomez A, Ferreira HJ, Bell JT, Sayols S, et al. DNA methylation profiling in breast cancer discordant identical twins identifies DOK7 as novel epigenetic biomarker. Carcinogenesis. 2013;34:102-8.

25. Falcon S, Gentleman R. Using GOstats to test gene lists for GO term association. Bioinformatics. 2007;23:257-8.

26. Crujeiras AB, Diaz-Lagares A, Sandoval J, Milagro Fl, Navas-Carretero S, Carreira MC, et al. DNA methylation map in circulating leukocytes mirrors subcutaneous adipose tissue methylation pattern: a genome-wide analysis from non-obese and obese patients. Sci Rep. 2017;7:41903.

27. Ronn T, Volkov P, Gillberg L, Kokosar M, Perfilyev A, Jacobsen AL, et al. Impact of age, BMI and $\mathrm{HbA} 1 \mathrm{c}$ levels on the genome-wide DNA methylation and mRNA expression patterns in human adipose tissue and identification of epigenetic biomarkers in blood. Hum Mol Genet. 2015; 24(13):3792-813.

28. Dick KJ, Nelson CP, Tsaprouni L, Sandling JK, Aïssi D, Wahl S, et al. DNA methylation and body-mass index: a genome-wide analysis. Lancet. 2014; 383(9933):1990-8.

29. Nicoletti CF, de Oliveira BA, de Pinhel MA, Donati B, Marchini JS, Salgado Junior $W$, et al. Influence of excess weight loss and weight regain on biochemical indicators during a 4-year follow-up after roux-en-Y gastric bypass. Obes Surg. 2015;25:279-84

30. Pinhel MA, Nicoletti CF, de Oliveira BA, Chaves RC, Parreiras LT, Sivieri T, et al. Weight loss and metabolic outcomes 12 months after roux-En-Y gastric bypass in a population of southeastern Brazil. Nutr Hosp. 2015;32:1017-21.

31. Nilsson EK, Ernst B, Voisin S, Almen MS, Benedict C, Mwinyi J, et al. Roux-en Y gastric bypass surgery induces genome-wide promoter-specific changes in DNA methylation in whole blood of obese patients. PLoS One. 2015;10:e0115186.

32. Benton MC, Johnstone A, Eccles D, Harmon B, Hayes MT, Lea RA, et al., An analysis of DNA methylation in human adipose tissue reveals differential modification of obesity genes before and after gastric bypass and weight loss, Genome Biol 2015; 16: 18.

33. Dahlman I, Sinha I, Gao H, Brodin D, Thorell A, Ryden M, et al. The fat cell epigenetic signature in post-obese women is characterized by global hypomethylation and differential DNA methylation of adipogenesis genes. Int J Obes. 2015;39:910-9.

34. Barres R, Kirchner H, Rasmussen M, Yan J, Kantor FR, Krook A, et al. Weight loss after gastric bypass surgery in human obesity remodels promoter methylation. Cell Rep. 2013;3:1020-7.

35. Kawai M, Mushiake S, Bessho K, Murakami M, Namba N, Kokubu C, et al. Wnt/Lrp/beta-catenin signaling suppresses adipogenesis by inhibiting mutual activation of PPARgamma and C/EBPalpha. Biochem Biophys Res Commun. 2007:363:276-82

36. Gustafson B, Gogg S, Hedjazifar S, Jenndahl L, Hammarstedt A, Smith U. Inflammation and impaired adipogenesis in hypertrophic obesity in man. Am J Physiol Endocrinol Metab. 2009;297:E999-E1003.

37. Wang X, Zhao X, Gao X, Mei Y, Wu M. A new role of p53 in regulating lipid metabolism. J Mol Cell Biol. 2013;5:147-50.

38. Minamino T, Orimo M, Shimizu I, Kunieda T, Yokoyama M, Ito T, et al. A crucial role for adipose tissue p53 in the regulation of insulin resistance. Nat Med. 2009;15:1082-7.

39. Morcillo S, Macías-González M, Tinahones FJ. The effect of metabolic and bariatric surgery on DNA methylation patterns. Curr Atheroscler Rep. 2017;19(10):40.

40. Garruti G, Di Ciaula A, Wang HH, Wang DQH, Portincasa P. Cross-talk between bile acids and gastro-intestinal and thermogenic hormones: clues from bariatric surgery. Annal of Hepatol. 2017;16 (Suppl. 1:s68-82.

41. Martín-Núñez GM, Cabrera-Mulero A, Alcaide-Torres J, García-Fuentes E, Tinahones FJ, Morcillo S. No effect of different bariatric surgery procedures on LINE-1 DNA methylation in diabetic and nondiabetic morbidly obese patients. Surg Obes Relat Dis. 2017;13(3):442-50.

42. Coppedè F, Seghieri M, Stoccoro A, Santini E, Giannini L, Rossi C, et al. DNA methylation of genes regulating appetite and prediction of weight loss after bariatric surgery in obese individuals. J Endocrinol Investig. 2019;42(1):37-44.

43. Nicoletti CF, Nonino CB, de Oliveira BA, Pinhel MA, Mansego ML, Milagro FI, et al. DNA methylation and Hydroxymethylation levels in relation to two weight loss strategies: energy-restricted diet or bariatric surgery. Obes Surg. 2016;26(3):603-11.

44. Paran CW, Verkerke AR, Heden TD, Park S, Zou K, Lawson HA, et al. Reduced efficiency of sarcolipin-dependent respiration in myocytes from humans with severe obesity. Obesity (Silver Spring). 2015;23:1440-9.

45. Nicoletti CF, Cortes-Oliveira C, Pinhel MAS, Nonino CB. Bariatric surgery and precision nutrition. Nutrients. 2017;9:974.

\section{Ready to submit your research? Choose BMC and benefit from:}

- fast, convenient online submission

- thorough peer review by experienced researchers in your field

- rapid publication on acceptance

- support for research data, including large and complex data types

- gold Open Access which fosters wider collaboration and increased citations

- maximum visibility for your research: over $100 \mathrm{M}$ website views per year

At BMC, research is always in progress.

Learn more biomedcentral.com/submissions 